\title{
Berberine Inhibits the Apoptosis-Induced
}

\section{Metastasis by Suppressing the iPLA2/LOX-5/LTB4 Pathway in Hepatocellular Carcinoma}

This article was published in the following Dove Press journal: OncoTargets and Therapy

\author{
Yawei Zhao',* \\ Kan $\mathrm{He}^{\mathrm{l}, *}$ \\ Huilin Zheng' \\ Madi Sun' \\ Tongfei Shi ${ }^{1}$ \\ Xiao Zheng' \\ Dan Shao ${ }^{1,2}$ \\ Hansi Zhang' \\ Fengying Guan' \\ Jing $\mathrm{Li}^{1}$ \\ Li Chen ${ }^{1,3}$
}

'Department of Pharmacology, College of Basic Medical Sciences, Jilin University, Changchun 13002I, People's Republic of China; ${ }^{2}$ Department of Biomedical Engineering, Columbia University, New York, NY 10027, USA; ${ }^{3}$ School of Nursing, Jilin University, Changchun 130020, People's Republic of China

*These authors contributed equally to this work
Correspondence: Jing Li

Department of Pharmacology, Basic

College of Medicine, Jilin University,

Changchun I3002I, People's Republic of

China

Tel/Fax+86 43I 85619799

Email lijing@jlu.edu.cn
Purpose: Hepatocellular carcinoma (HCC) is one of the most malignant cancers around the world. HCC is less sensitive to conventional cytotoxic agents and easily develops into systemic metastases. However, the molecular mechanisms of the metastasis of HCC are poorly understood and need elucidation.

Materials and Methods: Transwell system of the chemotherapy-challenged and unchallenged HepG2 cells was established. Adhesion assay and scratch-wound assay were utilized to analyze the adhesion and migration of HepG2 cells. iPLA2 and LOX-5 expression were analyzed by Western blot. LTB4 level was analyzed by ELISA.

Results: Chemotherapeutics are traditionally regarded as a way of killing tumor cells; on the other hand, we proved that the chemotherapeutics-induced tumor cell apoptosis can also change the tumor microenvironment by activating the LOX pathway and subsequently release inflammatory factors such as LTB4 which can stimulate the adhesion and migration of the small number of surviving cells. Berberine can reverse the adhesion and migration of HepG2 cells by inhibiting the expression of LOX-5 and reducing the LTB4 production in the tumor microenvironment.

Conclusion: Our study sheds light on a novel anti-metastasis strategy that the combination of Berberine and chemotherapy may prevent the chemotherapy-induced metastasis in HCC. Keywords: hepatocellular carcinoma, Berberine, chemotherapy, metastasis, LOX-5

\section{Introduction}

Hepatocellular carcinoma (HCC) is the third leading cause of cancer death around the world. ${ }^{1}$ At present, surgical removal of tumors, liver transplantation, radiotherapy and chemotherapy are the mainstay therapeutic strategy for HCC. ${ }^{2}$ However, the long-term survival in HCC patients after surgery remains less satisfactory, since HCC is sometimes resistant to conventional cytotoxic chemotherapeutics and is more likely to metastasize due to the rich vascular supply in liver. ${ }^{3}$ Evidence has suggested that complex biological and pathological events were involved in the process of HCC metastasis. ${ }^{4}$ Despite extensive efforts in clinical and basic research, the molecular mechanism of the chemotherapy-associated metastasis in HCC is poorly understood. Therefore, the investigation of new metastatic mechanisms and novel therapeutic strategies against the chemotherapy-induced metastasis in HCC is urgently needed.

Most common chemotherapeutics cause apoptosis in the targeted cancer cell. Although activation of the caspase 3 is a hallmark of apoptosis, it has been reported that the chemotherapy-induced of caspase 3 cleavage activates the cytosolic calcium- 
independent phospholipase A2 ( $\left.\mathrm{PLA}_{2}\right)$, and $\mathrm{PLA}_{2}$ triggers the cascade reaction of arachidonic acid (AA) metabolic pathway, which may be a stimulation of tumor growth. ${ }^{5-9}$ Cyclooxygenase (COX) pathway and Lipoxygenase (LOX) pathway are two major metabolism pathways of AA cascade. $^{10,11}$ Despite the massive studies of the COX pathway in malignant cancer, ${ }^{12-15}$ studies focusing on the LOX pathway remain rather inadequate. There is evidence that LOX plays an important role in tumor adhesion and metastasis. ${ }^{16}$ LOX was reported as the marker for predicting the likelihood of metastases to the bone in breast cancer patients. ${ }^{17}$ Several studies indicated the potential of inhibiting the LOX-5 in suppressing tumor cell proliferation in various cancers. ${ }^{18,19}$ Since Leukotriene B4 (LTB4) is one of the major end-products of the AA metabolism via the LOX pathway, the involvement of LTB4 in various carcinogenesis has also been indicated. ${ }^{20}$ Thus, there may be a possibility that the chemotherapeuticsinduced apoptosis may lead to the release of various inflammatory factors that change the microenvironment of cancer cells and promote the invasion and metastasis in HCC.

Berberine is an isoquinoline alkaloid that is found in the roots, stem bark and rhizome of the Berberis species including Rhizoma coptidis (Huanglian), Cortex phellodendri (Huang bai) and Hydrastis canadensis (goldenseal). ${ }^{8,21}$ Berberine exhibits multiple pharmacological activities such as anticancer and anti-diabetes mellitus. ${ }^{22,23}$ Our previous study found that Berberine inhibited the AA pathway by suppressing cPLA2 and COX-2 gene expressions in HCC in vivo and in vitro. ${ }^{21}$ However, although LOX is one of the key enzymes of the AA metabolic pathway, the effect of Berberine on the LOX pathway still remains unclear. In our study, we explored whether Berberine can overcome the chemotherapy-induced metastasis of liver cancer cells by inhibiting the LOX pathway.

In our study, we proved that the chemotherapeuticsinduced tumor cell apoptosis changed the tumor microenvironment by activating the LOX pathway. The increased secretion of inflammatory factors such as LTB4 ultimately stimulated the adhesion and migration of a small number of surviving tumor cells. And Berberine could reverse the adhesion and migration of HepG2 cells by inhibiting iPLA 2 and LOX-5 expression and reducing the LTB4 level in the tumor microenvironment. Our study sheds light on a novel anti-metastasis strategy that the combination of Berberine and chemotherapy may prevent the chemotherapy-induced metastasis in HCC.

\section{Materials and Methods}

\section{Chemicals and Reagents}

VP-16 (etoposide) injection was purchased from Qilu pharmaceutical Co., LTD. Berberine chloride hydrate $\left(\mathrm{C}_{20} \mathrm{H}_{18} \mathrm{ClNO}_{4}\right.$, Purity $>99 \%$, hereinafter referred to as Berberine) was kindly provided by the Northeast Pharmaceutical Group Co., Ltd. (Shenyang, China). All the chemical compounds were analytically pure reagents.

\section{Cell Culture}

The human hepatoma cell line HepG2 was purchased from The Cell Bank of the Chinese Academy of Sciences (Shanghai, China) and was cultured in RPMI 1640 supplemented with $10 \%$ fetal bovine serum (FBS). Cells were incubated at $37^{\circ} \mathrm{C}$ in $5 \% \mathrm{CO}_{2}$ and $95 \%$ air atmosphere and passed every 3 days.

\section{Establishment of the Transwell System and Migration Assay}

Transwell plates with $8.0 \mu \mathrm{m}$ pore polycarbonate membrane inserts (Cat\#3422, Corning) were used during the experiments. HepG2 cells $\left(5 \times 10^{4}\right.$ cells per well) were seeded into the 24 well plates and treated with $2.5 \mu \mathrm{M}$ VP-16 for $24 \mathrm{~h}$. Then, the culture medium was refreshed and the upper inserts with 1000 cells in $5 \mathrm{~mL}$ serum-free RPMI 1640 were placed into the wells daily during the 7 days' incubation. In parallel, after treatment with VP-16 (VP-16 group), $3.125 \mu$ M Berberine (VP-16 + Ber group) was added into the culture medium of the bottom wells and Berberine at the same concentration were added every 3 days. Fifty $\mu \mathrm{L}$ supernatants were collected every other day for the analysis of the LTB4 level. And the culture medium of the 7th day's incubation was collected for further experiment. The upper inserts with $1.5 \times 10^{5}$ cell $/ \mathrm{mL}$ in serum-free RPMI 1640 were placed into the wells on the 6th day of incubation. Cells from the outer side of the membrane were fixed and stained by $0.1 \%$ crystal violet for $20 \mathrm{~min}$ at room temperature after 18 $\mathrm{h}$ incubation. The cells were photographed under the microscope and counted, and the average number of cell in five visual fields was regarded as total cell numbers of migration.

\section{Western Blot Analysis}

The Western blot analysis was performed as previously described. ${ }^{9}$ Briefly, the cells after 5 days' incubation were collected for the Western blot analysis. The blots were blocked with $5 \%$ non-fat dried milk for $1 \mathrm{~h}$ at room temperature, and then incubated with anti-iPLA 2 (Cat. \# sc-25504, Santa Cruz Biotechnologies), anti-LOX-5 (Cat. 
\#sc-20785, Santa Cruz Biotechnologies) and anti-GAPDH (Cat. \# sc-25778, Santa Cruz Biotechnologies) antibodies (1:1000 diluted) overnight at $4^{\circ} \mathrm{C}$. Then, the membranes were incubated with HRP-conjugated secondary antibody (1:2000 diluted) for $2 \mathrm{~h}$ at room temperature.

\section{Measurements of LTB4 Levels}

The ELISA analysis was performed according to the manufacturer's instructions. Briefly, the collected supernatants (50 $\mu \mathrm{L} /$ test) from the Transwell co-culture system were thawed and LTB4 levels were analyzed by ELISA analysis using the Human LTB4 Parameter Assay Kit (Cat. \# KGE004B, R\&D Systems). The absorbance was read at a wavelength of $450 \mathrm{~nm}$.

\section{Scratch-Wound Assay}

Single-layer HCC cells were culture to grow confluence in 6-well plates, and then the wounds were made using a sterile 10 $\mu \mathrm{L}$ pipette tips. Cultures were then washed gently by PBS. Then, the cells were exposed to the culture medium of the 7th day's incubation of Berberine alone (Ber group), VP-16 alone (VP-16 group) or a combination of Berberine and VP-16 (Ber + VP-16 group) for migration. At least four different fields were photographed under the microscope at $0 \mathrm{~h}$ and $18 \mathrm{~h}$ after the scratch. Three wells for each group were used and three independent experiments were performed. Image $\mathrm{J}$ was used for analysis.

\section{Adhesion Assay}

To mimic the process of metastasis, the adhesion ability of HepG2 cells was evaluated. Cells $\left(2.5 \times 10^{5}\right.$ cells per well $)$ in the conditioned culture medium collected from the 7th day's incubation in the above groups were plated into 24 -well plates and shaken for $2 \mathrm{~h}$ at $37^{\circ} \mathrm{C}(150 \mathrm{rpm})$. The cells were photographed under the microscope. Moreover, adhesion ability between HepG2 cells and fibronectin (FN) was also analyzed. Ninety-six-well plates were coated with $10 \mu \mathrm{g} / \mathrm{mL}$ FN from human plasma (Cat. \# F8180, Solarbio), dried for $1-2 \mathrm{~h}$ and washed twice with PBS. The plate was then incubated for 2 $\mathrm{h}$ with $1 \%$ BSA (100 $\mu \mathrm{L}$ per well) in RPMI 1640 to block the nonspecific binding at $37^{\circ} \mathrm{C}$, and then rinsed twice. Cells $(4 \times$ $10^{4}$ per well) in culture medium collected from the 7th day's incubation were then added into the coated wells, and allowed to attach for $2 \mathrm{~h}$. Then, the cells were rinsed gently by PBS to remove the nonadherent cells and subsequently fixed with $10 \%$ TCA buffer for $1 \mathrm{~h}$ at $4^{\circ} \mathrm{C}$. The attached cells were stained with $4 \%$ SRB for $30 \mathrm{~min}$, followed by 5 washes with $1 \%$ acetic acid. The cells were lysed in 10mM Tris-base (PH 10.5) and the

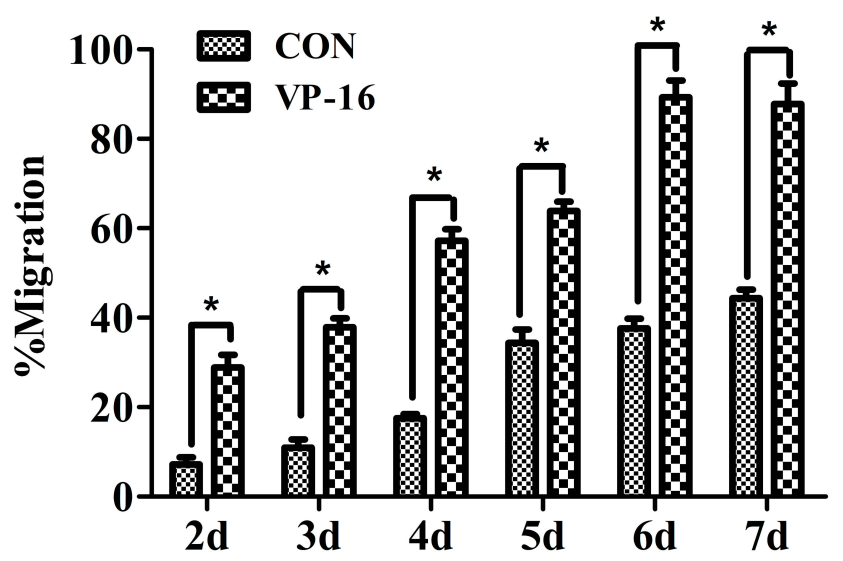

Figure I Migration of HepG2 cells was promoted by chemotherapy-induced apoptosis. After the cells on the bottom of the wells being treated with $2.5 \mu \mathrm{M}$ VP-16, crystal violet staining assay was applied to analyze the influence of the treated cells on the migration of HepG2 cells from day I to day 7. Results were expressed as the mean \pm SD of three independent experiments, ${ }^{*} p<0.05$.

released stain was quantified by absorbance at a wavelength of $540 \mathrm{~nm}$.

\section{Statistical Analysis}

The results were presented as the mean \pm SD. Statistical analyses were performed by GraphPad Prism. The significance of the difference between the two groups was assessed by the Student's $t$ test. The significance of the difference of multiple groups was analyzed of variance (ANOVA) with the NewmanKeuls tests. A value of $p<0.05$ was considered statistically significant.

\section{Results}

\section{Chemotherapy-Induced Apoptosis Promoted the Migration of Hepatoma Cells}

Tumor chemotherapy has been argued as a double-sided sword. On the one hand, a large number of tumor cells are killed, and tumor volume shrinks rapidly. On the other hand, it is demonstrated that chemotherapy may be an initial factor for recurrence and metastasis. In our study, co-culture system was designed using transwell chamber; the HepG2 cells in the upper insert chamber were cultured in the medium of HepG2 cells after chemotherapy in the bottom layer. As shown in Figure 1, the migration of HepG2 cells in VP-16 treated groups was all significantly higher than control and the increasement of migration rate was time dependent. These suggested that although chemotherapy played its part in killing a large amount of 
tumor cells, the change of the microenvironment caused by it may become a promoter of tumor cell migration.

\section{Berberine Reversed the}

Apoptosis-Induced Migration of HepG2

\section{Cells}

In the transwell system, consistent with the above results, VP-16 treatment significantly induced the migration of HepG2 cells compared with control. A nonapoptosis-causing concentration $(3.125 \mu \mathrm{M})$ of Berberine single treatment in both the upper and lower chambers did not show significant influence on cell migration in Berberine group while it partly abrogated the migration of cells in VP-16 + Berberine group (Figure 2A and B). Similar results were observed in scratch-wound assay: wounds in VP-16 group were significantly closer than those in control group, while the addition of Berberine could reverse the migration induced by VP-16 treatment (Figure 2C and D). These results indicated that Berberine reversed the migration of HepG2 cells induced by chemotherapy drug VP-16 challenge.

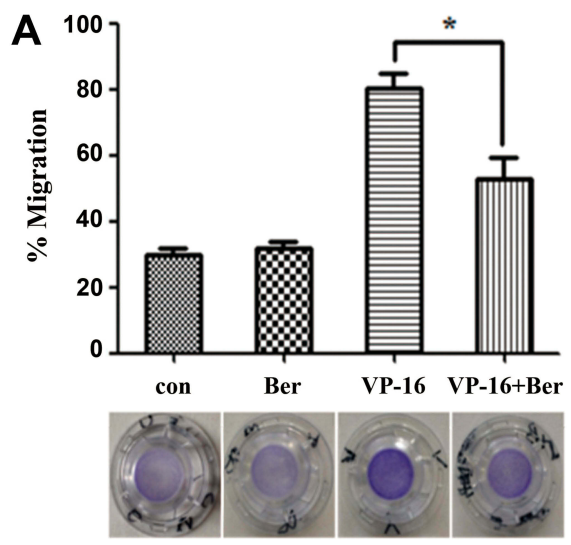

B

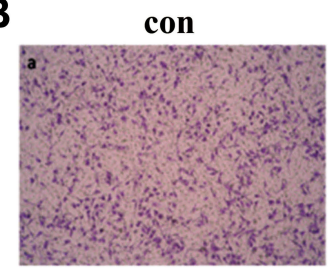

Ber

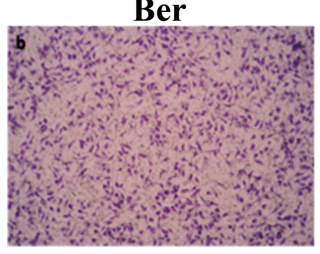

VP-16
VP-16

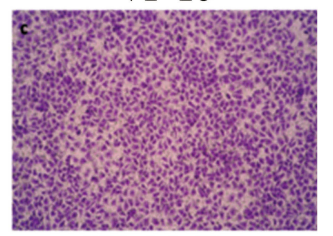

VP-16+Ber

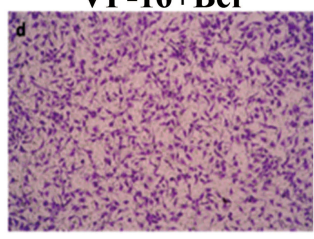

VP-16+Ber

C
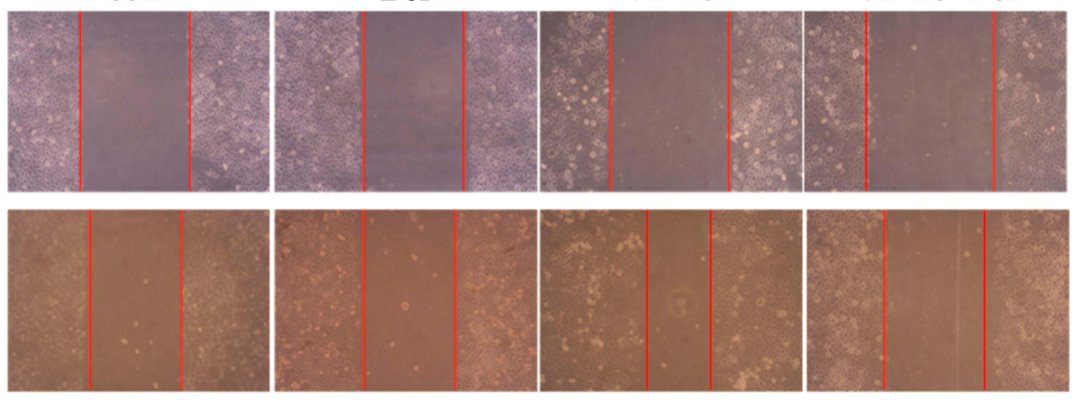

D

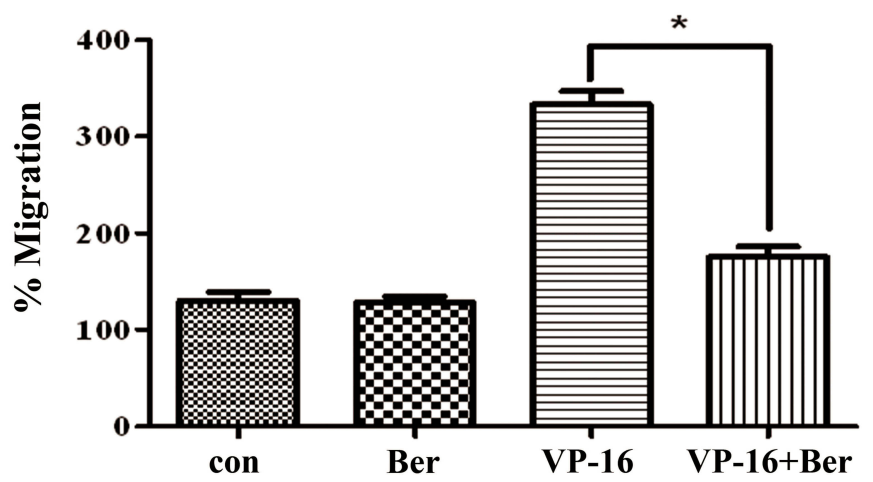

Figure 2 Berberine reversed the apoptosis-induced migration of HepG2 cells. (A) After the cells on the bottom of the wells being treated with $2.5 \mu \mathrm{M}$ VP-I6, $3.125 \mu \mathrm{M}$ Berberine was applied to the system and cultured for 6 days. Crystal violet staining assay was applied to analyze the migration of HepG2 cells. Cell numbers were counted under the microscope. (B) Cells from the outer side of the membrane were photographed under the microscope. (C) The cells were then exposed to the culture medium of the 7th day's incubation for the Scratch-wound assay. (D) Migration of the cells was analyzed by Image J. Each bar represents the mean \pm SD of three independent experiments, $n=3,{ }^{*}<0.05$. 


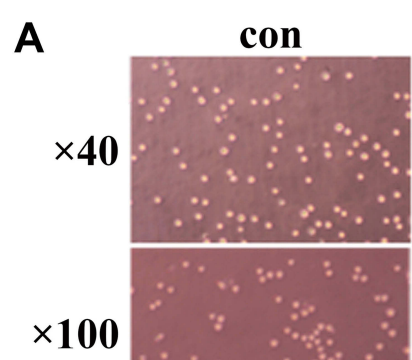

B

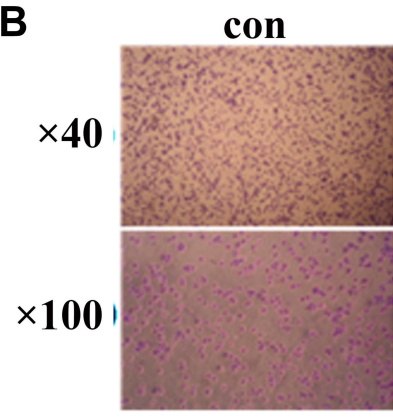

C

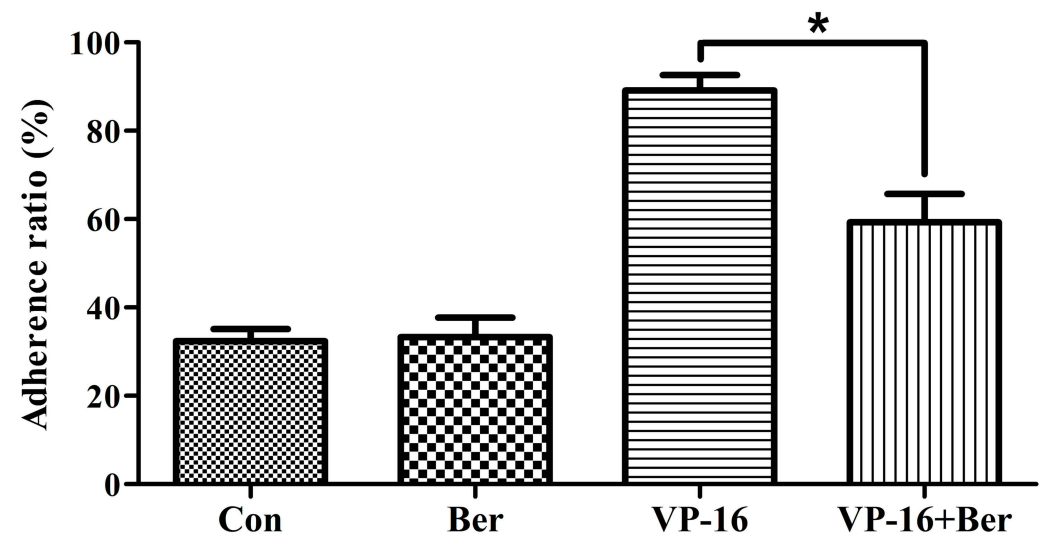

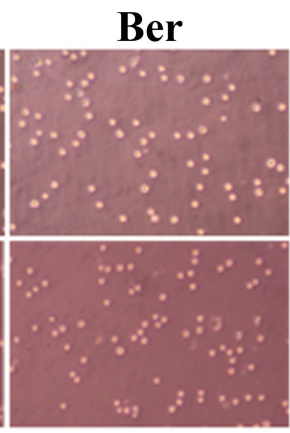

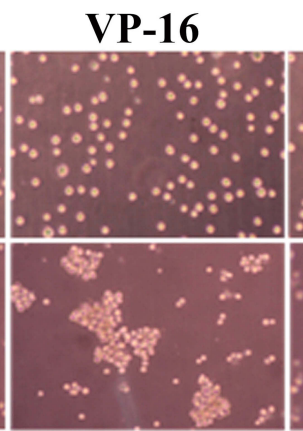

VP-16+Ber

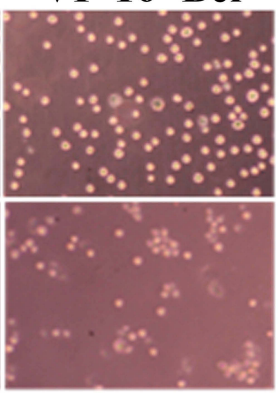

Ber
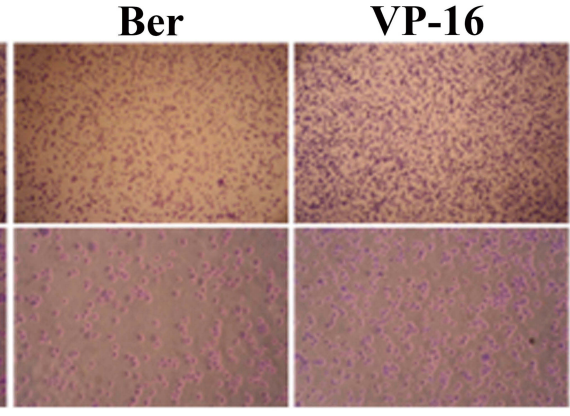

VP-16+Ber
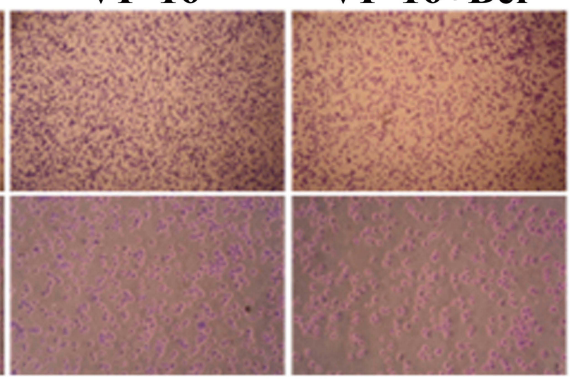

VP-16

Figure 3 Berberine reversed the apoptosis-induced Adhesion of HepG2 cells. (A) Cells were cultured in the culture medium collected from the 7th day's incubation and shaken for $2 \mathrm{~h}$ at $37^{\circ} \mathrm{C}$. (B) Cells in culture medium collected from the 7th day's incubation were added to the FN-coated wells, and allowed to attach for $2 \mathrm{~h}$. Attached cells were fixed, stained with $4 \%$ SRB. (C) stained cells were lysed in $10 \mathrm{mM}$ Tris-base and the released stain was quantified by absorbance at a wavelength of 540 nm. Each bar represents the mean $\pm S D$ of three independent experiments, $n=3,{ }^{*}<0.05$.

\section{Berberine Reversed the}

\section{Apoptosis-Induced Adhesion of HepG2 Cells}

Cell adhesion assay was conducted to analyze if chemotherapy treatment could promote the cell-cell or the cell-matrix adhesion. Cells were cultured in the culture medium of the 7th day's incubation of each group. VP16 treatment significantly induced the cell-cell adhesion of HepG2 cells compared with control while $3.125 \mu \mathrm{M}$ Berberine single treatment did not show a significant influence on the cell-cell adhesion in Berberine group. However, the same concentration of it greatly abrogated the adhesion induced by VP-16 treatment in VP16 +
Berberine group (Figure 3A). And as shown in Figure $3 \mathrm{~B}$ and $\mathrm{C}$, Berberine single treatment showed no significant influence on the cell adhesion to $\mathrm{FN}$ in Berberine group, while Berberine showed the ability to reverse the VP-16 treatment-induced cell-FN adhesion of HepG2 cells.

\section{Berberine Blocked the iPLA $2 / L O X-5 /$ LTB4 Pathway Activated by \\ Chemotherapy}

Phospholipids of cell membranes can be stimulated by $\mathrm{iPLA}_{2}$ and release AA, which can be ultimately converted by specific synthases including LOX-5 into LTB4. The 


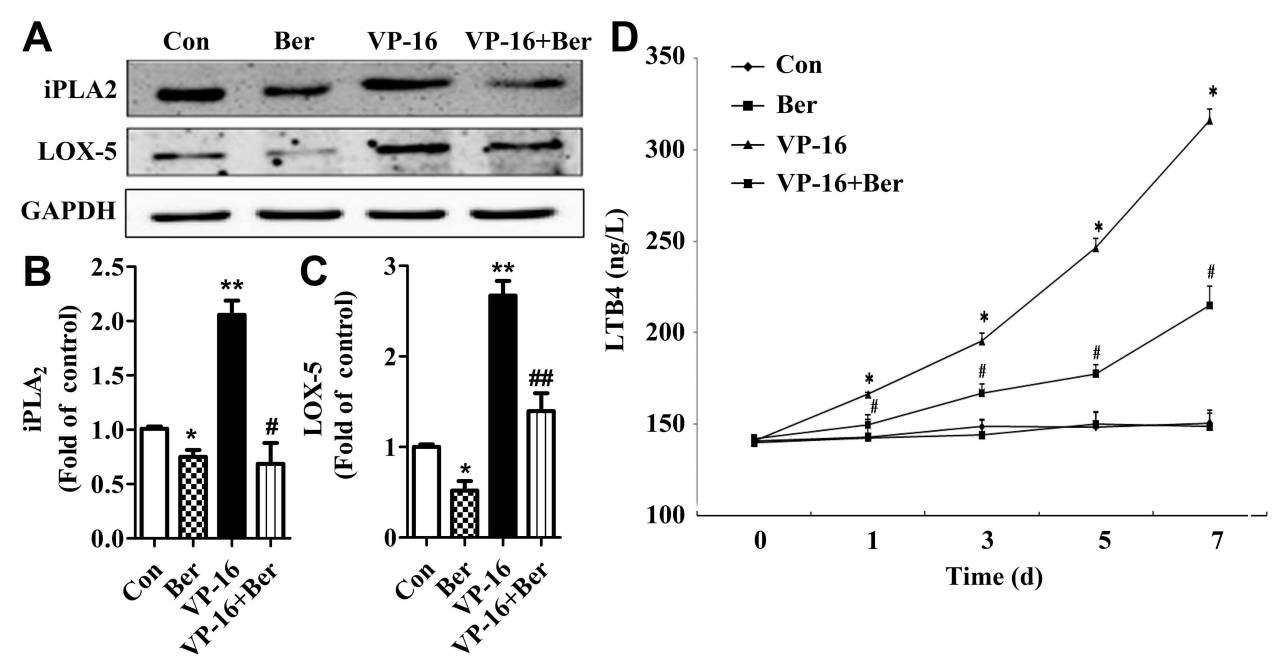

Figure 4 The protein expression of iPLA $A_{2}$ and LOX-5 in HepG2 cells treated by VP-16 and/or Berberine. (A) The cells after 5 days' incubation were collected and the protein expression of $\mathrm{PLA}_{2}$ and LOX-5 in HepG2 cells were measured by Western blot analysis. (B) Relative quantification of iPLA 2 levels expressed relative to control. (C) Relative quantification of LOX-5 levels expressed relative to control. (D) The LTB4 levels in HepG2 cells treated by VP-16 and/or Berberine. The supernatants of each group were collected each day during the 7 days' incubation. The LTB4 levels were measured by ELISA. Each bar represents the mean \pm SD of three independent experiments, $n=3$, ${ }^{*} \mathrm{p}<0.05$ compared with control; **p $<0.01$ compared with control; ${ }^{*} \mathrm{P}<0.05$ compared with VP-I6 group. ${ }^{\#} \mathrm{p}<0.0 \mathrm{I}$ compared with VP-I6 group.

expression of $\mathrm{iPLA}_{2}$ and LOX-5, the two key enzymes of the AA pathway can be inhibited by Berberine (Figure 4A-C). As VP-16 treatment showed significant activation of the two proteins, the combination of Berberine reversed this activation. In parallel, the change of LTB4 secretion in the supernatant in the 7 days' incubation was measured by ELISA (Figure 4D). The level of LTB4 time-dependently increased by solely VP-16 treatment, and Berberine could partly reverse the increase of LTB4 production compared with the VP-16 group in a time-dependent manner. These results indicated that Berberine reversed the chemotherapy-induced $\mathrm{PLA}_{2}-\mathrm{AA}$ /LOX-5/LTB4 pathway activation in HepG2 cells.

\section{Discussion}

Although being a mainstream of current cancer treatment with high rates of overall response, chemotherapy is now being questioned for its effect other than cytotoxicity. ${ }^{9}$ Recent reports suggested that chemotherapy-induced apoptotic cells could trigger the growth signals which stimulated the proliferation of the progenitor or stem cells. $^{24}$ The cleavage of caspases 3 and 7 activated ${ }_{i P L A}$ to trigger the AA cascade which leads to the production of the growth signal Prostaglandin E2 $\left(\mathrm{PGE}_{2}\right){ }^{6}$ The executioner caspases of apoptotic cells function as promoting tissue regeneration and wound healing, and the pathway was first named as "Phoenix Rising"

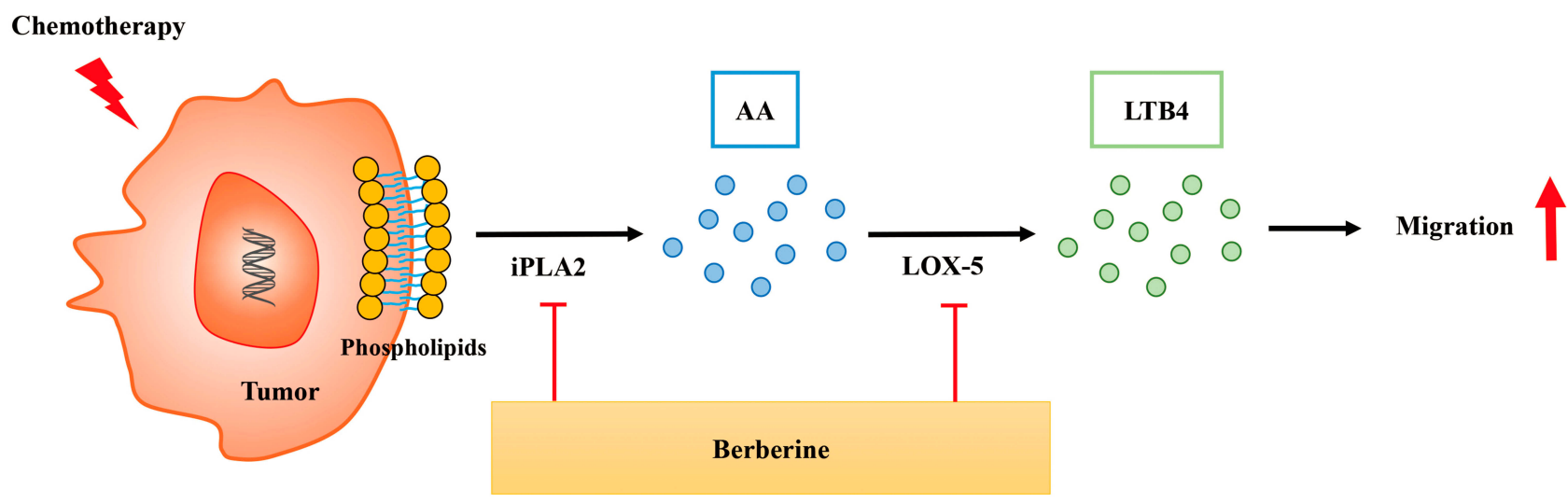

Figure 5 Schematic illustration of the mechanism by which Berberine inhibits the apoptosis-induced metastasis by suppressing the AA-LOX-5 pathway. Chemotherapeuticsinduced tumor cell apoptosis can activate the LOX pathway, and subsequently release inflammatory factor LTB4 which ultimately stimulates the adhesion and migration of the small number of surviving tumor cells. And Berberine can reverse the adhesion and migration by inhibiting the expression of iPLA ${ }_{2}$ and LOX-5 and reduce LTB4 production in the tumor microenvironment. 
pathway. ${ }^{6,7}$ A similar phenomenon was found in cancer cells. After the chemotherapy challenge, the few surviving cells can repopulate the chemotherapy-damaged tumor rapidly by the proliferation at an accelerated pace. ${ }^{8}$ Given the influence of the AA pathway in stimulating tumor repopulation, it probably exhibits a similar influence on the metastasis of HCC.

And relative to the widely studied $\mathrm{AA}-\mathrm{COX}-\mathrm{PGE}_{2}$ axis, ${ }^{9,10}$ the LOX pathway which is another important part of the AA pathway is less reported. LOX expression was found to be up-regulated in metastatic tumors and the inhibition of LOX suppressed metastasis in vivo ${ }^{25}$ Also LOX expression was indicated to be associated with the bone relapse and metastasis in ER-positive patients. ${ }^{16}$ Our result confirmed that the AA/LOX-5/LTB4 axis played a key role in chemotherapy-induced metastasis in the surviving HepG2 cells.

In our study, we focused on the blockage of the AA pathway in order to reverse the microenvironment changed by chemotherapy treatment. And we found that Berberine as an extraction from traditional Chinese herbs exhibited a strong ability on blocking the AA pathway by inhibiting LOX-5 and reduce the level of LTB4. Since the "Phoenix Rising" pathway demonstrated that the caspase- 3 mediated activation of the AA pathway played a key role in cancer progression, blocking this pathway may provide a promising strategy on cancer treatment and preventing cancer metastasis. Indeed, caspase 3 inhibition was reported to inhibit the proliferation of tumor cells in the experimental mice model. zDEVD-fmk (a caspase-3 inhibitor) was found to inhibit the proliferation of some tumors in combination with chemotherapeutics. ${ }^{22,23}$ However, the inhibition of caspase- 3 may reduce the efficiency of chemotherapy-induced the apoptosis. Therefore, targeting another key molecular in the "Phoenix Rising" pathway may be a promising strategy to overcome the chemotherapy-induced metastasis. Together with our previous studies, ${ }^{8,9,21}$ we found that Berberine being a dual inhibitor of $\mathrm{iPLA}_{2}$ and LOX-5, which are the two key enzymes of the AA pathway, can efficiently block the AA pathway without interfering with the apoptosis process. And this indicated the beneficial role of the combination of Berberine and chemotherapy in cancer treatment and metastasis prevention.

In conclusion, Berberine contributed to the inhibition of the chemotherapy challenge-induced metastasis in surviving liver cancer cells. Berberine suppressed the two rate-limiting enzymes iPLA2 and LOX-5 in the dying cells which contributed to the production of LTB4 into tumor microenvironment in HCC (Figure 5). Our work provided a promising strategy that the combination of Berberine and cytotoxic chemotherapeutics may suppress the progression and prevent the metastasis of liver cancer.

\section{Disclosure}

The authors have declared that no competing interest exists.

\section{References}

1. Tao YM, Liu Z, Liu HL. Dickkopf-1 (DKK1) promotes invasion and metastasis of hepatocellular carcinoma. Dig Liver Dis. 2013;45 (3):251-257. doi:10.1016/j.dld.2012.10.020

2. Zhang Y, Shi ZL, Yang X, Yin ZF. Targeting of circulating hepatocellular carcinoma cells to prevent postoperative recurrence and metastasis. World $J$ Gastroenterol. 2014;20(1):142-147. doi:10.3748/wjg.v20.i1.142

3. Yang Y, Nagano H, Ota $\mathrm{H}$, et al. Patterns and clinicopathologic features of extrahepatic recurrence of hepatocellular carcinoma after curative resection. Surgery. 2007;141(2):196-202. doi:10.1016/j. surg.2006.06.033

4. Wang L, Yao M, Dong Z, Zhang Y, Yao D. Circulating specific biomarkers in diagnosis of hepatocellular carcinoma and its metastasis monitoring. Tumour Biol. 2014;35(1):9-20. doi:10.1007/ s13277-013-1141-0

5. Huang Q, Li F, Liu X, et al. Caspase 3-mediated stimulation of tumor cell repopulation during cancer radiotherapy. Nat Med. 2011;17 (7):860-866. doi: $10.1038 / \mathrm{nm} .2385$

6. Li F, Huang Q, Chen J, et al. Apoptotic cells activate the "phoenix rising" pathway to promote wound healing and tissue regeneration. Sci Signal. 2010;3(110):ra13. doi:10.1126/scisignal.2000634

7. Yom SS. Accelerated repopulation as a cause of radiation treatment failure in non-small cell lung cancer: review of current data and future clinical strategies. Semin Radiat Oncol. 2015;25(2):93-99. doi:10.1016/j.semradonc.2014.12.002

8. Zhao Y, Cui L, Pan Y, et al. Berberine inhibits the chemotherapy-induced repopulation by suppressing the arachidonic acid metabolic pathway and phosphorylation of FAK in ovarian cancer. Cell Prolif. 2017;50(6):e12393. doi:10.1111/cpr.12393

9. Cui L, Zhao Y, Pan Y, et al. Chemotherapy induces ovarian cancer cell repopulation through the caspase 3-mediated arachidonic acid metabolic pathway. Onco Targets Ther. 2017;10:5817-5826. doi:10.2147/OTT.S150456

10. Liaras K, Fesatidou M, Geronikaki A. Thiazoles and thiazolidinones as COX/LOX inhibitors. Molecules. 2018;23(3):685.

11. P JJ, Manju SL, Ethiraj KR, Elias G. Safer anti-inflammatory therapy through dual COX-2/5-LOX inhibitors: a structure-based approach. Eur J Pharm Sci. 2018;121:356-381. doi:10.1016/j.ejps.2018.06.003

12. Fisher JC, Gander JW, Haley MJ, et al. Inhibition of cyclo-oxygenase 2 reduces tumor metastasis and inflammatory signaling during blockade of vascular endothelial growth factor. Vasc Cell. 2011;3(1):22. doi:10.1186/2045-824X-3-22

13. Su JL, Shih JY, Yen ML, et al. Cyclooxygenase-2 induces EP1- and HER-2/Neu-dependent vascular endothelial growth factor-C up-regulation: a novel mechanism of lymphangiogenesis in lung adenocarcinoma. Cancer Res. 2004;64(2):554-564. doi:10.1158/ 0008-5472.CAN-03-1301

14. Koehne CH, Dubois RN. COX-2 inhibition and colorectal cancer. Semin Oncol. 2004;31(2 Suppl 7):12-21. doi:10.1053/j.seminoncol. 2004.03.041 
15. Mandal P, Kundu BK, Vyas K, et al. Ruthenium(ii) arene NSAID complexes: inhibition of cyclooxygenase and antiproliferative activity against cancer cell lines. Dalton Trans. 2018;47(2):517-527. doi:10.1039/C7DT03637J

16. Cox TR, Gartland A, Erler JT. Lysyl oxidase, a targetable secreted molecule involved in cancer metastasis. Cancer Res. 2016;76 (2):188-192. doi:10.1158/0008-5472.CAN-15-2306

17. Cox TR, Rumney RMH, Schoof EM, et al. The hypoxic cancer secretome induces pre-metastatic bone lesions through lysyl oxidase. Nature. 2015;522(7554):106-110. doi:10.1038/nature14492

18. Matsuyama M, Yoshimura R, Tsuchida K, et al. Lipoxygenase inhibitors prevent urological cancer cell growth. Int J Mol Med. 2004;13 (5):665-668

19. Tong WG, Ding XZ, Witt RC, Adrian TE. Lipoxygenase inhibitors attenuate growth of human pancreatic cancer xenografts and induce apoptosis through the mitochondrial pathway. Mol Cancer Ther. 2002;1(11):929-935.

20. Ihara A, Wada K, Yoneda M, Fujisawa N, Takahashi H, Nakajima A. Blockade of leukotriene B4 signaling pathway induces apoptosis and suppresses cell proliferation in colon cancer. J Pharmacol Sci. 2007;103(1):24-32. doi:10.1254/jphs.FP0060651
21. Li J, Li O, Kan M, et al. Berberine induces apoptosis by suppressing the arachidonic acid metabolic pathway in hepatocellular carcinoma. Mol Med Rep. 2015;12(3):4572-4577. doi:10.3892/mmr.2015.3926

22. Teschendorf P, Vogel P, Wippel A, et al. The effect of intracerebroventricular application of the caspase-3 inhibitor zDEVD-FMK on neurological outcome and neuronal cell death after global cerebral ischaemia due to cardiac arrest in rats. Resuscitation. 2008;78 (1):85-91. doi:10.1016/j.resuscitation.2008.02.010

23. Gdynia G, Grund K, Eckert A, et al. Basal caspase activity promotes migration and invasiveness in glioblastoma cells. Mol Cancer Res. 2007;5(12):1232-1240. doi:10.1158/1541-7786.MCR-07-0343

24. Zhao Y, He M, Cui L, et al. Chemotherapy exacerbates ovarian cancer cell migration and cancer stem cell-like characteristics through GLI1. Br J Cancer. 2020. doi:10.1038/s41416-020-0825-7

25. Miller BW, Morton JP, Pinese M, et al. Targeting the LOX/hypoxia axis reverses many of the features that make pancreatic cancer deadly: inhibition of LOX abrogates metastasis and enhances drug efficacy. EMBO Mol Med. 2015;7(8):1063-1076. doi:10.15252/ emmm.201404827

\section{Publish your work in this journal}

OncoTargets and Therapy is an international, peer-reviewed, open access journal focusing on the pathological basis of all cancers, potential targets for therapy and treatment protocols employed to improve the management of cancer patients. The journal also focuses on the impact of management programs and new therapeutic agents and protocols on patient perspectives such as quality of life, adherence and satisfaction. The manuscript management system is completely online and includes a very quick and fair peer-review system, which is all easy to use. Visit http://www.dovepress.com/ testimonials.php to read real quotes from published authors. 